Gynäkologe 2010 • 43:975-976

DOI 10.1007/s00129-010-2640-3

Online publiziert: 28. November 2010

(c) Springer-Verlag 2010

\author{
W. Jonat ${ }^{1} \cdot$ R. Kreienberg ${ }^{2} \cdot$ W. Janni ${ }^{3} \cdot$ C. Mundhenke ${ }^{1}$ \\ ${ }^{1}$ Klinik für Gynäkologie und Geburtshilfe, Universität Schleswig-Holstein, \\ Campus Kiel \\ 2 Universitätsfrauenklinik und Poliklinik, Ulm \\ ${ }^{3}$ Universitätsfrauenklinik, Heinrich-Heine-Universität Düsseldorf
}

\title{
Mammakarzinom - neue Aspekte aus Praxis und Politik
}

man daher die Nebenwirkungen aggressiver Therapien ersparen kann. Einen aktuellen Überblick über genexpressionsbasierte Risikoscores und histopathologische Marker gibt der Beitrag von Herrn PD Dr. Rody.

Im operativen Management des Mammakarzinoms ist viel über die Morbidität, aber auch den klinischen Nutzen einer Axilladissektion bei cNo und günstiger Risikokonstellation gesprochen worden. Die Wächterlymphknotenbiopsie ist mittlerweile Standard bei den meisten cNo-Konstellationen. Beim diesjährigen ASCO-Kongress wurde darüber hinaus diskutiert, ob ein klinischer Nutzen für eine Axilladissektion nach positivem Sentinel-Lymphknotenstatus gegeben ist oder ob auch dann auf eine weitergehende Operation verzichtet werden kann. Herr Dr. Schem gibt in seinem Beitrag einen Überblick über die aktuelle Datenlage.

Status, ergänzt, sodass zielgerichtete Therapien für Subkollektive möglich werden. Seit einigen Jahren werden zusätzliche prädiktive Marker diskutiert, sie finden zunehmend auch in Deutschland Eingang in die Klinik. Unter anderem werden Gensignaturen entwickelt, welche die Prognose und besonders das Therapieansprechen besser abzuschätzen helfen sollen. Zurzeit werden sie in großen, prospektiven Studien validiert. Durch die Gensignaturen könnten Patientengruppen identifiziert werden, die von bestimmten Chemotherapien nicht profitieren und denen divrisiko besteht (z. B. höheres Lebensalter, hormonsensibler Tumor und No), so dass eine Radiatio keine Verbesserung der Ausgangslage mit sich brächte. Diese Frage ist insofern von größter Aktualität, als sich schon heute (und zukünftig noch weiter zunehmend) ein Großteil unserer Patientinnen in der Altersgruppe über 70 Lebensjahren befindet.

Die endokrine Therapie des Mammakarzinoms war vermutlich die erste zielgerichtete Therapie. Als zweiter tumorbiologischer Marker hat die Her-2/neuÜberexpression entscheidende prognostische und therapeutische Bedeutung erlangt. Die aggressiv wachsenden Mammakarzinome mit HER-2-Überexpression kommen infrage für eine zielgerichtete Therapie mit dem monoklonalen Antikörper Trastuzumab, der schon heute in der adjuvanten und in der metastasierten Situation zu deutlich höheren Heilungsbzw. PFS (progressionsfreies Überleben)Raten beiträgt. Mittlerweile ist in der metastasierten Situation (und HER-2/neuÜberexpression) mit Lapatinib auch ein Tyrosinkinaseinhibitor (in Kombination mit Capecitabin) nach Trastuzumab-Versagen zugelassen. Sein Stellenwert in der adjuvanten und auch in der metastasierten Situation wird zurzeit in klinischen Studien weiter untersucht.

Etliche weitere neue tumorbiologische Marker werden klinisch als geeignete Ziele einer selektiven Therapie überprüft. Besonders interessant in diesem Zusam- 
menhang sind Wachstumsrezeptoren, intrazelluläre Signalwege sowie Proliferations- und Apoptosemarker. Darüber hinaus stehen Antiangiogenesesubstanzen zur Verfügung, die gezielt Endothelzellen der Tumorblutgefäße ausschalten und dem Tumor das Nährstoffangebot entziehen. Die erste für die metastasierte Situation zugelassene Substanz ist Bevacizumab. Einen Überblick über den Stellenwert der antikörper- und inhibitorbasierten Therapien gibt Herr PD Dr. Herr.

Da momentan auch zur zielgerichteten Therapie der tripelnegativen Tumoren geforscht wird, ist der Beitrag von Frau Dr. Hüttemann zum Stellenwert der BRCA-Gene beim tripelnegativen Mammakarzinom besonders wichtig.

\section{() Diskussionen zur}

Kosteneffizienz sollten von Medizinern selbst angestoßen und geführt werden

Die zielgerichteten systemischen Therapien bedingen eine deutliche Erhöhung der Therapiekosten. In Deutschland befinden sich die Diskussionen über die Kosteneffizienz von Diagnose- und Therapieverfahren noch in den Anfängen. Daher ist es wichtig, den Blick auf das westliche Ausland zu werfen und zu betrachten, wie dort Kosteneffizienzanalysen für zielgerichtete Substanzen erstellt werden. Die Ergebnisse sind nicht direkt übertragbar, sie geben jedoch Hinweise auf die Größenordnung der Kosten und die Effizienzbewertung. Diese Erläuterungen werden von Prof. C. Mundhenke dargestellt.

Die Individualisierung der medikamentösen, operativen und strahlentherapeutischen Behandlung bei Mammakarzinom schreitet zügig voran. Da die Ressourcen auch im deutschen Gesundheitssystem limitiert sind, müssen sich zunehmend alle neuen Optionen nicht nur Analysen zur klinischen Effektivität, sondern auch Analysen zur Kosteneffizienz stellen. Diese durchaus auch gesamtgesellschaftlich zu führenden Diskussionen sollten von den Medizinern selbst angestoßen und geführt werden, denn andernfalls werden die Entscheidungen von fachfremden Politikern und Verwaltungen getroffen.

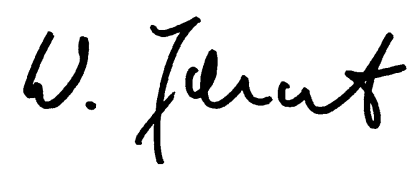

Prof. Dr. W. Jonat, Kiel
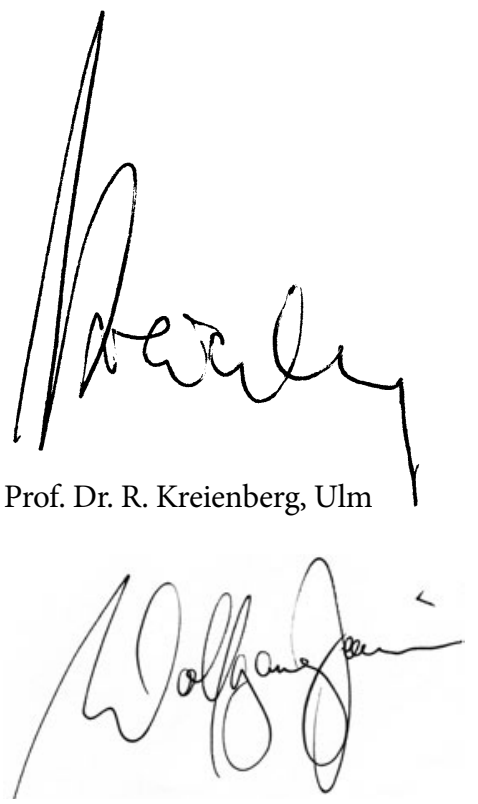

Prof. Dr. W. Janni, Düsseldorf

\section{Korrespondenzadresse}

\section{Prof. Dr. W. Jonat}

Klinik für Gynäkologie und Geburtshilfe, Universität Schleswig-Holstein, Campus Kiel Michaelisstr. 16, 24105 Kiel

jonat@email.uni-kiel.de
Der Gynäkologe bietet jeden Monat umfassende und aktuelle Beiträge zu interessanten Themenschwerpunkten aus allen Bereichen der Gynäkologie und Geburtshilfe.

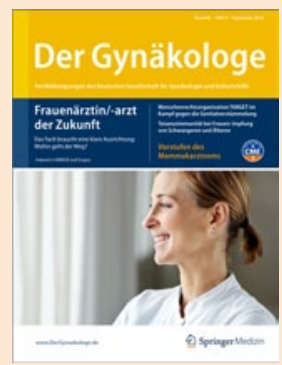

\section{Leitthemen in} Der Gynäkologe 2011

- 01/11 Kontrazeption

- 02/11 Möglichkeiten und Grenzen der Reproduktionsmedizin

- 03/11 Die gynäkologisch-geburtshilfliche OP-Einheit

- 04/11 Genetik

- 05/11 Rechtliche Auflagen in Klinik und Praxis

- 06/11 Frauenärztlich-pädiatrische Kooperationen I

- 07/11 Gerinnungsstörungen in der Geburtshilfe

- 08/11 Virusinfektionen der Schwangeren

- 09/11 Update Gyn Onko: Ovar, Vulva, Endometrium

- 10/11 Ökonomische Rahmenbedingungen der Frauenheilkunde

- 04/11 Neurologische Erkrankungen und SS

- 04/12 Prävention und Betreuung der übergewichtigen Patientin

(Änderungen vorbehalten)

So erreichen Sie unseren Kundenservice:

Springer Medizin Verlag GmbH Kundenservice Zeitschriften

Haberstraße 7

69126 Heidelberg

Tel. +496221 345-4303

Fax: +49 $6221345-4229$

E-Mail: Leserservice@springer.com

www.DerGynaekologe.de 